\title{
Genetic Background Behind the Amino Acid Profiles of Fermented Soybeans Produced by Four Bacillus spp.
}

\author{
Mihyun Jang $^{1 \dagger}$, Do-Won Jeong ${ }^{2 \dagger}$, Ganghun Heo ${ }^{1}$, Haram Kong ${ }^{1}$, Cheong-Tae Kim ${ }^{3}$, and \\ Jong-Hoon Lee ${ }^{1 *}$ \\ ${ }^{1}$ Department of Food Science and Biotechnology, Kyonggi University, Suwon 16227, Republic of Korea \\ ${ }^{2}$ Department of Food and Nutrition, Dongduk Women's University, Seoul 02748, Republic of Korea \\ ${ }^{3}$ Nongshim Co., Ltd., Seoul 07065, Republic of Korea
}

\begin{abstract}
Strains of four Bacillus spp. were respectively inoculated into sterilized soybeans and the free amino acid profiles of the resulting cultures were analyzed to discern their metabolic traits. After 30 days of culture, B. licheniformis showed the highest production of serine, threonine, and glutamic acid; B. subtilis exhibited the highest production of alanine, asparagine, glycine, leucine, proline, tryptophan, and lysine. $B$. velezensis increased the $\gamma$-aminobutyric acid (GABA) concentration to $>\mathbf{2 0 0} \%$ of that in the control samples. B. sonorensis produced a somewhat similar amino acid profile with B. licheniformis. Comparative genomic analysis of the four Bacillus strains and the genetic profiles of the produced free amino acids revealed that genes involved in glutamate and arginine metabolism were not common to the four strains. The genes gadA/B (encoding a glutamate decarboxylase), rocE (amino acid permease), and puuD ( $\gamma$-glutamyl- $\gamma$-aminobutyrate hydrolase) determined GABA production, and their presence was species-specific. Taken together, $B$. licheniformis and $B$. velezensis were respectively shown to have high potential to increase concentrations of glutamic acid and GABA, while $B$. subtilis has the ability to increase essential amino acid concentrations in fermented soybean foods.
\end{abstract}

Keywords: Soybean fermentation, Bacillus, $\gamma$-aminobutyric acid, gadA/B, rocE, puuD

Received: December 30, 2020 Accepted: January 21, 2021

First published online: January 22, 2021

*Corresponding author Phone: +82-31-249-9656 Email:jhl@kgu.ac.kr

${ }^{\dagger}$ These authors contributed equally to this work.

pISSN 1017-7825 elSSN 1738-8872

Copyright(C) 2021 by The Korean Society for Microbiology and Biotechnology

\section{Introduction}

Several types of fermented soybean food are consumed in Korea and representative examples are ganjang (soy sauce) and doenjang (soybean paste). The traditional production of ganjang and doenjang starts from the manufacture of meju. Meju is made by soaking, steaming, crushing, and molding soybeans into blocks, followed by spontaneous fermentation for 2-3 months. The ripened meju is mixed with brine and ripened for a further 2-3 months, then the liquid portion of the mixture is separated, resulting in a traditional type of ganjang. The remaining solid portion is subsequently mashed and fermented for $>6$ months and becomes quality doenjang [1]. Ripened meju is used as a starter culture as well as a nutrient and flavor source for fermented food production in Korea [2].

Understanding of traditional fermented soybean foods has long been a research theme of food scientists in Korea. Several studies including microbial community analysis have provided basic insight for accelerated ripening, quality assurance, and flavor enhancement of fermented soybean foods. Early microbial studies isolated and identified microorganisms exhibiting strong amylase, protease, and lipase activities that can contribute to degradation of soybean macromolecules [3-6]. More recent culture-independent microbial community analysis techniques have revealed the presence of a wider variety of microorganisms in the food matrices [7-15]. However, regardless of the analytical methods used, most microbial studies reported that the bacterial genus Bacillus and the fungal genus Aspergillus were the most populous microorganisms.

Recently, molecular biology techniques and sequence databases have contributed to identification, characterization, and typing of bacteria and increased the number of novel bacterial species [16]. Rapid advances in whole-genome sequencing technologies and analysis pipelines have further enhanced the resolution power of bacterial species and influenced the taxonomic status of closely related identities. This advanced bacterial identification methodology has affected the consolidation and assignment of new taxa in the genus Bacillus [1719]. In this context, we isolated and identified Bacillus spp. from 12 meju samples collected from five regions of Korea to determine the predominant Bacillus species in meju based on current bacterial taxonomic standards [20]. One hundred and fifty-one Bacillus isolates were identified, in the following order of predominance: B. velezensis, B. sonorensis, B. subtilis, and B. licheniformis. 
The safety-approved Aspergillus oryzae is normally used as a starter in the production of commercial fermented soybean foods in Korea. Several studies have employed Bacillus spp. in the soybean food manufacturing process, and the selected starters reportedly accomplished their target purposes [21-26], but Bacillus spp. have not yet been introduced in mass production. A lack of detailed understanding of the contribution of each Bacillus species to soybean food fermentation hinders the introduction of Bacillus spp. as starter cultures.

In the current study, we respectively inoculated one strain each of B. velezensis, B. sonorensis, B. subtilis, and B. licheniformis into sterilized soybeans and analyzed the free amino acid profiles in the soybean culture samples during fermentation to discern their metabolic traits and provide information for selecting Bacillus starter culture candidates for soybean food fermentation. We also performed a comparative genomic analysis of the four Bacillus strains to shed light on the genetic profiles of the free amino acids they produce.

\section{Materials and Methods \\ Bacillus Strains and Cultures}

All selected Bacillus strains were isolated from fermented soybean foods or soybeans and their complete genome sequences have been published (Table 1). B. licheniformis 14ADL4 (KCTC 33983) was isolated in our laboratory and deposited in the Korean Collection for Type Cultures [27]. B. sonorensis SRCM101395 was kindly provided by the Microbial Institute for Fermentation Industry (http://mifi.kr). B. subtilis ATCC 21228 (KCCM 40443) was purchased from the Korean Culture Center of Microorganisms (http://kccm.or.kr/). B. velezensis JJD34 was kindly provided by Prof. Che Ok Jeon, Chung-Ang University, South Korea [28]. All Bacillus strains were cultured on Difco tryptic soy agar (BD Diagnostic Systems, USA) and in Difco tryptic soy broth (TSB; BD Diagnostic Systems) at $37^{\circ} \mathrm{C}$ for $24 \mathrm{~h}$.

\section{Preparation of Bacillus Strain-Inoculated Soybean Samples}

Korean soybeans, known as baektae (Glycine max L. Merrill), were washed, soaked in equal amounts of water for $18 \mathrm{~h}$ at room temperature, and then crushed using a mortar. Fifty grams of the crushed soybeans was placed in $500-\mathrm{ml} \mathrm{reagent} \mathrm{bottles} \mathrm{then} \mathrm{autoclaved} \mathrm{for} 30 \mathrm{~min}$ at $121^{\circ} \mathrm{C}$. Respective logarithmic-phase Bacillus cells cultured in TSB were inoculated into the crushed and sterilized soybeans at $5 \times 10^{5}$ colony-forming units (CFU)/g then mixed thoroughly. Samples were prepared in duplicate and incubated aseptically at $25^{\circ} \mathrm{C}$ for 30 days along with crushed and sterilized soybean samples as controls. This culture temperature was set to align with future production of fermented soybean foods at room temperature. Samples were collected at days 1, 15, and 30 and stored at $-70^{\circ} \mathrm{C}$ for subsequent chemical and microbiological analyses.

\section{Viable Cell Count and pH Analysis of Soybean Culture Samples}

Five grams of each sample was homogenized with $20 \mathrm{ml}$ sterilized peptone water and filtered through sterilized cheesecloth. The filtrates were spread onto Difco plate count agar (BD Diagnostic Systems) after serial dilution using saline then incubated at $37^{\circ} \mathrm{C}$ for $24 \mathrm{~h}$ to determine viable cell numbers. The $\mathrm{pH}$ of the filtrates was measured using a pH meter. All experiments were conducted on three independent samples prepared in the same way.

\section{Analysis of Free Amino Acids in Soybean Culture Samples}

Samples ( $5 \mathrm{~g}$ ) were mixed with $50 \mathrm{ml}$ of $75 \%$ aqueous $\mathrm{EtOH}(\mathrm{v} / \mathrm{v})$. The mixture was sonicated for $1 \mathrm{~h}$, soaked at room temperature for $24 \mathrm{~h}$, and then filtered through a $0.2-\mu \mathrm{m}$ membrane filter (Phenomenex, USA). The filtrate was analyzed by a custom service provided by the National Instrumentation Center for Environmental Management in Korea (http://nicem.snu.ac.kr/). The analyses were performed using the Dionex Ultimate 3000 HPLC system (Thermo Scientific, USA). Chromatographic separation was achieved with a VDSpher $100 \mathrm{C} 18$-E column $(150 \times 4.6 \mathrm{~mm}, 3.5 \mu \mathrm{m}$; VDS Optilab, Germany). Gradient elution was carried out with sodium phosphate

Table 1. Genomic features of Bacillus strains used in this study.

\begin{tabular}{lllll}
\hline \multicolumn{1}{c}{ Strain } & \multicolumn{1}{c}{$\begin{array}{c}\text { B. licheniformis } \\
\text { 14ADL4 }\end{array}$} & $\begin{array}{c}\text { B. sonorensis } \\
\text { SRCM101395 }\end{array}$ & $\begin{array}{c}\text { B. subtilis ATCC } \\
\text { 21228 }\end{array}$ & $\begin{array}{c}\text { B. velezensis } \\
\text { JJ-D34 }\end{array}$ \\
\hline Chromosome size (bp) & $4,332,232$ & $4,832,293$ & $4,141,030$ & $4,105,955$ \\
Chromosome G+C content (\%) & 45.9 & 45.2 & 43.5 & 46.2 \\
No. of plasmids & - & - & 1 & - \\
Plasmid size (bp) & - & - & 85,618 & - \\
Plasmid G+C content (\%) & - & - & 38.1 & - \\
Predicted CDSs & 4,273 & 4,609 & 4,151 & 3,907 \\
No. of rRNAs & 24 & 24 & 30 & 87 \\
No. of tRNAs & 81 & 85 & 27 & 1 \\
Contigs & 1 & 1 & Soybeans & Doenjang \\
Origin & Doenjang & Food & USA & Korea \\
Country & Korea & Korea & NZ_CP020023.1, & NZ_CP011346.1 \\
Accession No. & NZ_CP026673.1 & NZ_CP021920.1 & NZ_CP020024.1 & {$[28]$} \\
Reference & [27] & - & - & \\
\hline
\end{tabular}

Genomic features were referred from analysis results in the NCBI database. 
buffer (solvent A; pH 7) and water/acetonitrile/methanol (solvent B; 10:45:45, v/v/v). The following binary mobile phase linear gradients were used: $100 \% \mathrm{~A}$ at $0 \mathrm{~min}, 95 \% \mathrm{~A}$ at $24 \mathrm{~min}, 45 \% \mathrm{~A}$ at $25 \mathrm{~min}, 20 \% \mathrm{~A}$ at $34.5 \mathrm{~min}$, and $95 \%$ $\mathrm{A}$ at $35 \mathrm{~min}$. The column temperature and flow rate were $40^{\circ} \mathrm{C}$ and $1 \mathrm{ml} / \mathrm{min}$, respectively. The detection was performed using a fluorescent detector. Two derivatizing agents, OPA (o-phthaldialdehyde; Agilent, USA) and FMOC (9-fluorenylmethoxycarbonyl chloride; Agilent), were simultaneously used according to the manufacturer's instructions. Excitation/emission wavelengths were respectively $340 / 450 \mathrm{~nm}$ for OPA-derivatized amino acids and 266/305 nm for FMOC-derivatized amino acids. The concentrations of individual free amino acids were determined using five-point calibration curves of Amino Acid Standard (WAT088122, Waters Corporation, USA). The free amino acid content of two samples prepared in the same conditions was analyzed twice.

\section{Comparative Genomic Analysis and Metabolic Pathway Prediction of Bacillus Strains}

Genomic information for comparative genomic analyses of Bacillus strains including B. licheniformis 14ADL4, B. sonorensis SRCM 101395, B. subtilis ATCC 21228, and B. velezensis JJ-D34 was downloaded from the NCBI database (http://ncbi.nlm.nih.gov/genomes), and the EzBioCloud database (https://www.ezbiocloud.net/). Coregenome and pan-genome analyses were performed using the Efficient Database framework for comparative Genome Analyses using BLASTP score Ratios (EDGAR) [29]. Two genes were considered orthologous when a bidirectional best BLAST hit with a single score ratio value threshold of at least $32 \%$ was obtained for orthology estimation. The B. licheniformis 14ADL4 genome was used as the reference genome for Venn diagram construction for four-genome analysis using EDGAR. Rapid Annotation using Subsystem Technology (RAST) [30] and the Interactive Pathways Explorer v3 (https://pathways.embl.de/) were used to determine gene contents based on functional subsystem classifications and to estimate the amino acid metabolic pathways. Comparative analyses at the protein level were performed by an all-against-all comparison of the annotated genomes.

\section{Statistical Analysis}

One-way analysis of variance followed by Duncan's multiple range test was used to evaluate significant differences between the average values obtained in the free amino acid analyses. $p$-value $<0.05$ was considered statistically significant. To visualize the differences between the amino acids produced from the sterilized soybeans by the inoculated bacteria, principal component analysis (PCA) was applied with maximum variation rotation. All statistical analyses were performed using the SPSS software package (version 22.0; SPSS, IBM, USA).

\section{Results}

\section{Growth of Four Bacillus Strains in Soybean Cultures and Their Contribution to pH Changes}

Fewer than $10 \mathrm{CFU} / \mathrm{g}$ were detected in the control soybean samples at day 30 (Fig. 1A). Most microorganisms present in the soybeans were eliminated by autoclaving $\left(30 \mathrm{~min}\right.$ at $\left.121^{\circ} \mathrm{C}\right)$. The almost constant $\mathrm{pH}$ during the 30 day incubation of control soybean samples demonstrated that few changes occurred in these cultures (Fig. 1B).

In inoculated cultures, there was no distinguishable difference in the cell numbers of the Bacillus strains on day 1 (average cell number $2.00 \times 10^{7} \mathrm{CFU} / \mathrm{g}$ ). Until day $15, B$. subtilis and B. velezensis maintained consistent growth, but $B$. licheniformis and B. sonorensis did not. At day 30 , the number of $B$. velezensis cells $\left(1.02 \times 10^{9} \mathrm{CFU} / \mathrm{g}\right)$ was almost 100 times that of $B$. licheniformis $\left(1.10 \times 10^{7} \mathrm{CFU} / \mathrm{g}\right)$; those were the highest and lowest cell numbers in the soybean cultures. The growth rates of the Bacillus strains were not sufficient to assert that the highest growth on soybeans among the four Bacillus spp. was by B. velezensis. According to Jang et al. [20], B. velezensis was the most populous Bacillus species identified in meju, which provides evidence that $B$. velezensis grows better on soybeans than B. licheniformis.

As the fermentation progressed, the samples inoculated with Bacillus showed a tendency of $\mathrm{pH}$ increase and then decrease. The increase of $\mathrm{pH}$ until day 15 can be attributed to the amino acids and amines produced by the degradation of soybean protein, and the $\mathrm{pH}$ decrease after day 15 might be caused by acid production by the strains [31].

A

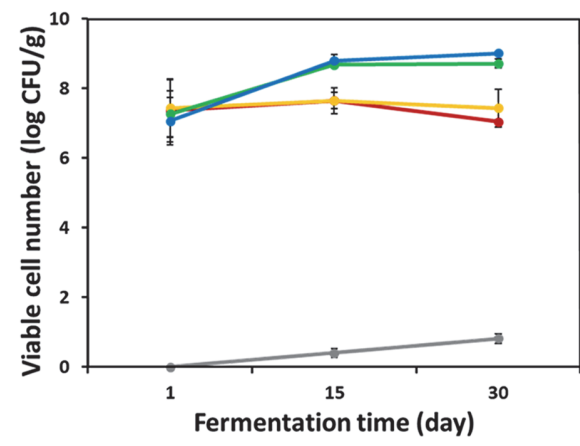

B

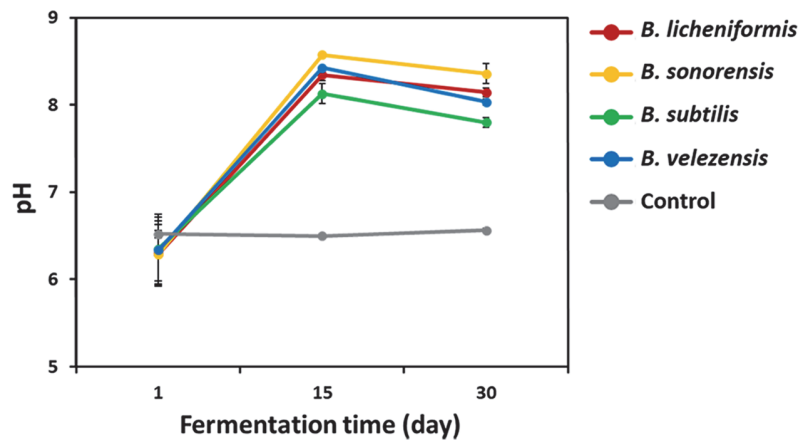

Fig. 1. Growth (A) and $\mathrm{pH}$ changes (B) of soybean cultures inoculated with four Bacillus strains over 30 days of incubation. 
Table 2. Free amino acid profiles in soybean cultures of four Bacillus spp. strains at days 1, 15, and 30.

(unit: $\mathrm{mg} / \mathrm{kg}$ )

\begin{tabular}{|c|c|c|c|c|c|c|c|c|c|c|c|c|c|c|c|}
\hline \multirow{2}{*}{ Amino acid } & \multicolumn{3}{|c|}{ Control } & \multicolumn{3}{|c|}{ B. licheniformis 14ADL4 } & \multicolumn{3}{|c|}{ B. sonorensis SRCM101395 } & \multicolumn{3}{|c|}{ B. subtilis ATCC 21228} & \multicolumn{3}{|c|}{ B. velezensis JJ-D34 } \\
\hline & Day 1 & Day 15 & Day 30 & Day 1 & Day 15 & Day 30 & Day 1 & Day 15 & Day 30 & Day 1 & Day 15 & Day 30 & Day 1 & Day 15 & Day 30 \\
\hline \multicolumn{16}{|c|}{ Neutral amino acid } \\
\hline Alanine & $160.67^{\mathrm{a}}$ & $9.17^{\mathrm{a}}$ & 191. & $67.67^{\mathrm{a}}$ & $433.26^{\mathrm{bc}}$ & $3049.71^{\mathrm{cd}}$ & $165.75^{\mathrm{a}}$ & $2034.47^{\mathrm{b}}$ & $2382.13^{\mathrm{bc}}$ & $158.93^{\mathrm{a}}$ & $3388.02^{\mathrm{d}}$ & $3799.28^{\mathrm{d}}$ & $167.77^{\mathrm{a}}$ & $752.88^{\mathrm{a}}$ & $937.89^{\mathrm{a}}$ \\
\hline Gly & $46.11^{\mathrm{a}}$ & $50.93^{\mathrm{a}}$ & $48.09^{\mathrm{a}}$ & $52.93^{\mathrm{a}}$ & $1172.65^{\mathrm{d}}$ & $1519.46^{\mathrm{e}}$ & $50.77^{\mathrm{a}}$ & $757.26^{\mathrm{bc}}$ & $960.31^{\mathrm{cd}}$ & $53.39^{\mathrm{a}}$ & $1970.79^{\mathrm{f}}$ & $2065.46^{\mathrm{f}}$ & $57.75^{\mathrm{a}}$ & $494.35^{\mathrm{b}}$ & $690.44^{\mathrm{bc}}$ \\
\hline Isoleucine & $.21^{\mathrm{a}}$ & $42.19^{\mathrm{a}}$ & $50.69^{\mathrm{a}}$ & $6.79^{\mathrm{a}}$ & $2767.39^{\mathrm{cd}}$ & $3318.77^{\mathrm{d}}$ & $47.77^{\mathrm{a}}$ & $1913.29^{\mathrm{b}}$ & $2386.80^{\mathrm{bc}}$ & $57.67^{\mathrm{a}}$ & $3155.71^{\mathrm{cd}}$ & $3562.05^{\mathrm{d}}$ & $64.04^{\mathrm{a}}$ & $3113.75^{\mathrm{cd}}$ & $3284.90^{\mathrm{d}}$ \\
\hline Leu & $5.26^{\mathrm{a}}$ & $55.82^{\mathrm{a}}$ & $67.84^{\mathrm{a}}$ & $74.86^{\mathrm{a}}$ & $5695.12^{\mathrm{cd}}$ & $6756.67^{\text {de }}$ & $73.82^{\mathrm{a}}$ & $4159.09^{b}$ & $4858.47^{b c}$ & $85.40^{\mathrm{a}}$ & $6710.01^{\mathrm{de}}$ & $7463.43^{\mathrm{e}}$ & $102.82^{\mathrm{a}}$ & $5299.35^{\mathrm{bc}}$ & $5494.63^{\text {cd }}$ \\
\hline Met & $21^{\mathrm{a}}$ & $19.35^{\mathrm{a}}$ & 21.9 & $.04^{\mathrm{a}}$ & 1150 & $1191.66^{\mathrm{ef}}$ & $28.80^{\mathrm{a}}$ & $860.04^{\mathrm{cd}}$ & $790.27^{b c}$ & $31.21^{\mathrm{a}}$ & 1286 & $950.86^{\text {cde }}$ & $32.97^{\mathrm{a}}$ & $1030.11^{\text {cde }}$ & f 526 . \\
\hline Phenyla & $79.03^{a}$ & $81.29^{\mathrm{a}}$ & $93.30^{\mathrm{a}}$ & $3.67^{\mathrm{a}}$ & $637.11^{\mathrm{cd}}$ & $5103.84^{\mathrm{d}}$ & $92.25^{\mathrm{a}}$ & $3540.35^{\mathrm{b}}$ & $3731.90^{\mathrm{bc}}$ & $107.18^{\mathrm{a}}$ & $5339.07^{\mathrm{d}}$ & $5745.45^{\mathrm{d}}$ & $119.31^{\mathrm{a}}$ & $5349.07^{\mathrm{d}}$ & $5285.23^{\mathrm{d}}$ \\
\hline Prol & $56.90^{\mathrm{a}}$ & $32.72^{\mathrm{a}}$ & $33.61^{\mathrm{a}}$ & $.88^{\mathrm{a}}$ & $2346.05^{\mathrm{b}}$ & $2958.76^{\mathrm{bc}}$ & $31.20^{\mathrm{a}}$ & $2947.26^{\mathrm{bc}}$ & $=3079.49^{b c}$ & $47.95^{\mathrm{a}}$ & $4016.13^{\mathrm{bc}}$ & $4227.06^{\mathrm{c}}$ & $58.32^{\mathrm{a}}$ & $3167.93^{b c}$ & $3739.25^{\mathrm{bc}}$ \\
\hline Seri & $44^{\mathrm{a}}$ & $52.83^{\mathrm{a}}$ & $61.08^{\mathrm{a}}$ & $.62^{\mathrm{a}}$ & $70^{\mathrm{e}}$ & $2006.15^{\mathrm{f}}$ & $57.23^{\mathrm{a}}$ & $1165.90^{\mathrm{d}}$ & $1468.59^{\mathrm{e}}$ & $74.70^{\mathrm{a}}$ & $753.50^{\mathrm{c}}$ & $1121.47^{\mathrm{d}}$ & $77.51^{\mathrm{a}}$ & $398.49^{b}$ & $690.07^{\mathrm{c}}$ \\
\hline Thr & $.51^{\mathrm{a}}$ & $49.16^{\mathrm{a}}$ & $60.11^{\mathrm{a}}$ & $53.44^{\mathrm{a}}$ & $2411.26^{\mathrm{f}}$ & $2434.95^{\mathrm{f}}$ & $50.72^{\mathrm{a}}$ & $1140.35^{\mathrm{de}}$ & $1340.80^{\mathrm{e}}$ & $71.26^{\mathrm{a}}$ & $886.79^{\mathrm{cd}}$ & $1092.44^{\mathrm{de}}$ & $75.19^{\mathrm{a}}$ & $434.53^{\mathrm{b}}$ & $648.53^{\mathrm{bc}}$ \\
\hline Tryl & $176.27^{\mathrm{a}}$ & $180.59^{\mathrm{a}}$ & $190.18^{a}$ & $194.04^{\mathrm{a}}$ & $3^{\mathrm{cd}}$ & $1325.69^{\mathrm{de}}$ & $196.15^{\mathrm{a}}$ & $763.09^{\mathrm{b}}$ & $832.84^{\mathrm{bc}}$ & $191.29^{\mathrm{a}}$ & $1825.04^{\mathrm{f}}$ & $1732.31^{\mathrm{ef}}$ & $208.42^{\mathrm{a}}$ & $1672.99^{\mathrm{ef}}$ & $1304.24^{\mathrm{de}}$ \\
\hline Tyr & $63.91^{\mathrm{a}}$ & $65.23^{\mathrm{a}}$ & $80.33^{\mathrm{a}}$ & $73.41^{\mathrm{a}}$ & $3213.56^{\mathrm{cd}}$ & $3280.44^{\mathrm{cd}}$ & $70.59^{\mathrm{a}}$ & $2439.95^{\mathrm{b}}$ & $2628.89^{b c}$ & $89.61^{a}$ & $3681.09^{\mathrm{d}}$ & $3218.17^{\mathrm{cd}}$ & $97.86^{\mathrm{a}}$ & $2993.13^{\mathrm{bcd}}$ & $2982.32^{\mathrm{bcd}}$ \\
\hline Valine & 54.58 & .74 & 6 & $.66^{\mathrm{a}}$ & bcd & 3977. & $66.21^{\mathrm{a}}$ & $2^{\mathrm{b}}$ & 298 & $78.99^{\mathrm{a}}$ & 4482 & 5062 & $89.25^{\mathrm{a}}$ & 439 & $4648.09^{\mathrm{e}}$ \\
\hline \multicolumn{16}{|c|}{ Acidic amino acid } \\
\hline Glutamic acid & & $410.35^{\mathrm{a}}$ & $434.01^{\mathrm{a}}$ & $420.44^{\mathrm{a}}$ & $18077.65^{\mathrm{e}}$ & $20053.26^{\mathrm{e}}$ & $415.27^{\mathrm{a}}$ & 10107.50 & ${ }^{c} 112$ & $445.43^{\mathrm{a}}$ & $12783.35^{\mathrm{cd}}$ & ${ }^{d} 14715.05^{d}$ & $472.16^{\mathrm{a}}$ & $5457.30^{\mathrm{b}}$ & $6723.10^{\mathrm{b}}$ \\
\hline \multicolumn{16}{|l|}{ Basic amino acid } \\
\hline Arginine & $57^{\mathrm{d}}$ & $1059.49^{\mathrm{d}}$ & 107 & 11 & 19 & 1 & & 32 & . & 103 & & $278.52^{\mathrm{bc}}$ & $1139.26^{\mathrm{d}}$ & 等 & $371.46^{\mathrm{c}}$ \\
\hline Histi & & $54.18^{\mathrm{a}}$ & $60.52^{\mathrm{a}}$ & $71.27^{\mathrm{a}}$ & & $1990.60^{\mathrm{cd}}$ & $57.10^{\mathrm{a}}$ & $1619.26^{\text {bc }}$ & $1290.84^{\mathrm{b}}$ & $63.51 \mathrm{a}^{\mathrm{a}}$ & $2136.37^{\mathrm{d}}$ & $1968.19^{\mathrm{cd}}$ & $74.30^{\mathrm{a}}$ & $2065.33^{\mathrm{cd}}$ & $1599.05^{\mathrm{bc}}$ \\
\hline Lysine & $73.71^{\mathrm{a}}$ & $85.49^{\mathrm{a}}$ & $95.88^{\mathrm{a}}$ & $93.60^{\mathrm{a}}$ & $5057.28^{\mathrm{cd}}$ & $6007.25^{\mathrm{de}}$ & $108.06^{\mathrm{a}}$ & $3823.86^{\mathrm{b}}$ & $4168.82^{\mathrm{bc}}$ & $122.86^{\mathrm{a}}$ & $6921.71^{\text {ef }}$ & $7272.85^{\mathrm{f}}$ & $134.86^{\mathrm{a}}$ & $4945.31^{\text {bcd }}$ & $4509.57^{\mathrm{bc}}$ \\
\hline \multicolumn{16}{|l|}{ Functiona } \\
\hline GABA & $232.27^{\mathrm{b}}$ & $257.63^{\mathrm{b}}$ & $285.51^{\mathrm{b}}$ & $273.72^{\mathrm{b}}$ & $40.36^{\mathrm{a}}$ & $49.14^{\mathrm{a}}$ & $275.59^{\mathrm{b}}$ & $53.89^{\mathrm{a}}$ & $60.93^{\mathrm{a}}$ & $303.79^{\mathrm{b}}$ & $270.59^{\mathrm{b}}$ & $309.81^{\mathrm{b}}$ & $316.22^{\mathrm{b}}$ & $668.28^{c}$ & $698.54^{\mathrm{c}}$ \\
\hline
\end{tabular}

Different superscripts within a row denote a significant difference between mean values $(p<0.05)$ according to Duncan's multiple range test.

\section{Free Amino Acid Production in Soybean Cultures by Four Bacillus Strains}

Nineteen free amino acids including $\gamma$-aminobutyric acid (GABA), a non-proteinogenic amino acid, were quantified in the controls and Bacillus strain-inoculated soybean cultures (Table 2). The concentration of free amino acids in the control samples did not change significantly during the 30-day incubation. Sixteen of the 18 identified proteinogenic amino acids were increased in concentration by the growth of the four Bacillus spp. A decrease in arginine concentration was detected in all of the inoculated soybean cultures, and a decrease of asparagine concentration was identified in B. sonorensis cultures.

After 30 days of culture, B. licheniformis showed the highest production of three amino acids (serine, threonine, and glutamic acid), and B. subtilis exhibited the highest production of seven amino acids (alanine, asparagine, glycine, leucine, proline, tryptophan, and lysine). Regardless of the inoculated Bacillus spp., the amount of glutamic acid produced from the soybean cultures was highest among the 19 amino acids. B. licheniformis was the highest producer, with a 47.7 -fold increase in glutamic acid concentration at day 30 compared with day 1 . The concentration of GABA in the soybean cultures was not significantly changed by $B$. subtilis. However, $B$. licheniformis and $B$. sonorensis decreased the GABA concentration in their soybean cultures to $<20 \%$ of that in the control samples after 15 days, while B. velezensis increased the GABA concentration to $>200 \%$ of that in the control samples in the same period. No correlation was found between the growth and the amino acid profiles produced by the four Bacillus spp. Free amino acid production by the four Bacillus spp. might depend on their metabolic traits rather than their biological activities.

PCA for Free Amino Acids Produced by the Four Bacillus Strains

Statistics on the 19 free amino acids produced from sterilized soybeans by the growth of four Bacillus spp. were subjected to PCA (Fig. 2). Arginine concentration decreased in the cultures of the four Bacillus spp. and this amino acid is located in the positive part of the PC1 dimension in the PCA factor loading plot, while the other 18 free amino acids were negatively correlated with PC1 (Fig. 2A). GABA increased only in the cultures of $B$. velezensis and is isolated in the PCA factor loading plot.

The PCA scores of the four Bacillus spp.-inoculated soybean cultures after 1, 15, and 30 days of incubation are shown in Fig. 2B. All factor scores at day 1 clustered together with those of the control samples, indicating that the amino acid production by Bacillus spp. in a short-term soybean culture was not sufficient to indicate the specific characteristics of the strains. The factor scores of Bacillus spp.-inoculated samples developed in different directions during incubation, but the factor scores on day 30 compared with day 15 do not exhibit the same dramatic differences as those on day 15 compared with day 1. Fifteen-day soybean cultures of the four Bacillus spp. may be sufficient to discern their specific characteristics in amino acid production.

The factor scores of $B$. licheniformis-inoculated samples developed in a similar direction as those of the B. sonorensis-inoculated samples. The production of alanine, serine, and threonine determined the direction of B. licheniformis- and B. sonorensis-inoculated samples in the factor score plot. B. licheniformis exhibited the highest production of serine and threonine and B. sonorensis was the next highest producer of both amino acids. The increase ratios of alanine by $B$. licheniformis and B. sonorensis were between those of B. subtilis and B. velezensis. 

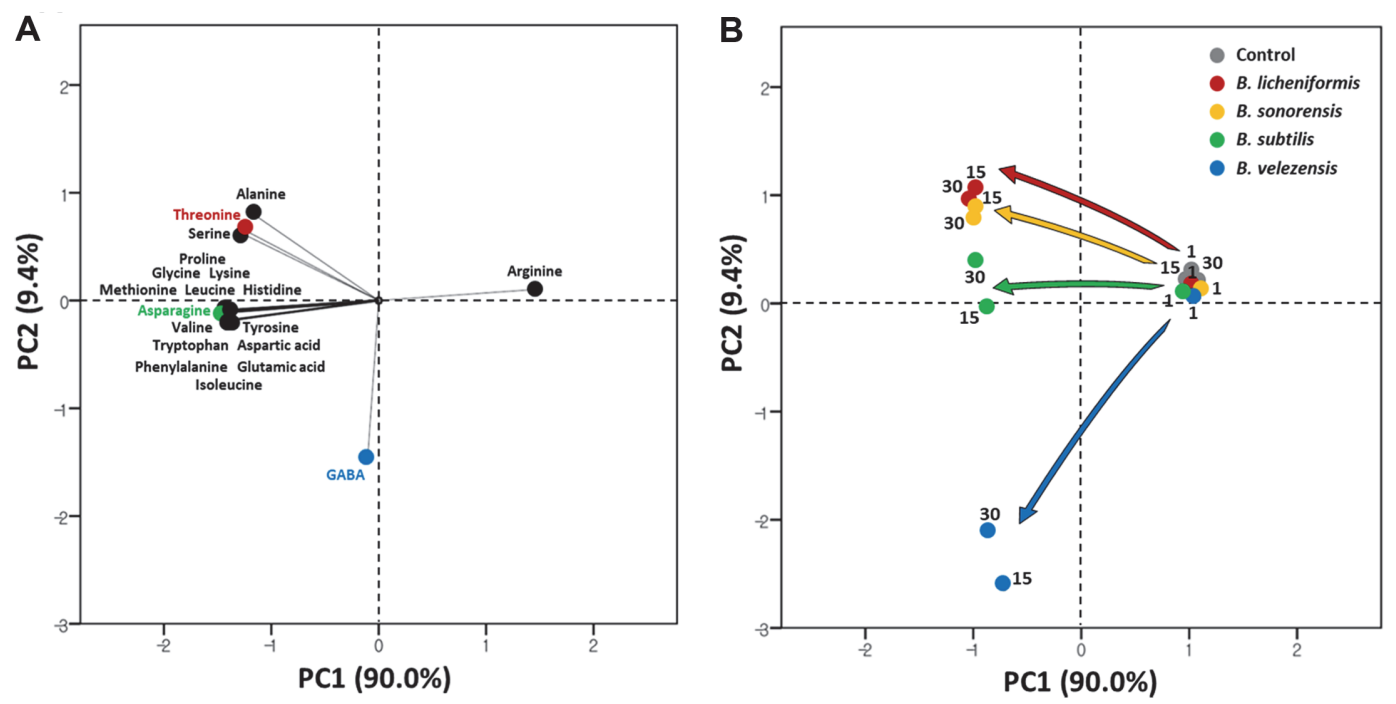

Fig. 2. Principal component analysis loadings from four Bacillus spp.-inoculated soybean samples over 30 days of incubation for (A) amino acid concentrations and (B) factor scores. Numbers indicate the incubation time of samples in days.

The B. licheniformis and B. sonorensis strains produced somewhat similar amino acid profiles in the soybean cultures. The production of 14 amino acids including the essential amino acids isoleucine, leucine, methionine, phenylalanine, tryptophan, valine, and lysine determined the direction of $B$. subtilis-inoculated samples in the factor score plot. The amount of asparagine produced by B. subtilis during the 30 -day-incubation was $>2.5$ times greater than that of the other strains. Asparagine may be the crucial amino acid determining the direction of the factor scores of B. subtilis. The production of GABA determined the locations of B. velezensis factor scores, which are distant from those of the other species. The profile differences of the amino acids produced by the four Bacillus strains instigated further studies to illuminate the determinants and characteristics involved in amino acid production dependent on species.

Genomic Insight into the Amino Acid Profiles of Soybean Cultures Produced by Four Bacillus Strains

The gene pools shared by the genomes of the four Bacillus strains are depicted in a Venn diagram (Fig. 3). These four strains share 2,486 protein-coding sequences (CDSs) in their core genome, corresponding to $53.9 \%-63.6 \%$ of their CDSs. The genome of B. velezensis JJ-D34 has $8.9 \%$ unique CDSs (i.e., ones that are absent from the other three Bacillus genomes). The proportions of unique CDSs in the genomes of B. licheniformis 14ADL4, B. sonorensis SRCM101395, and B. subtilis ATCC 21228 are $7.3 \%, 11.9 \%$, and $8.8 \%$, respectively.

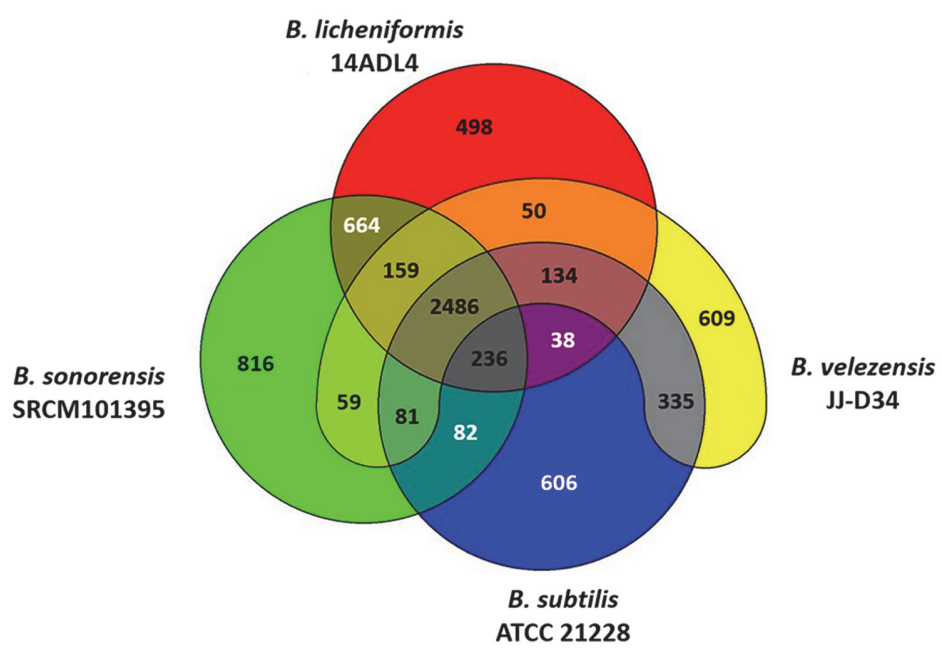

Fig. 3. Venn diagram showing the pan-genome of four Bacillus spp. strains. Overlapping regions represent common coding sequences (CDSs) shared between the genomes. The numbers outside the overlapping regions indicate the numbers of CDSs in each genome without homologs in the other genomes. 


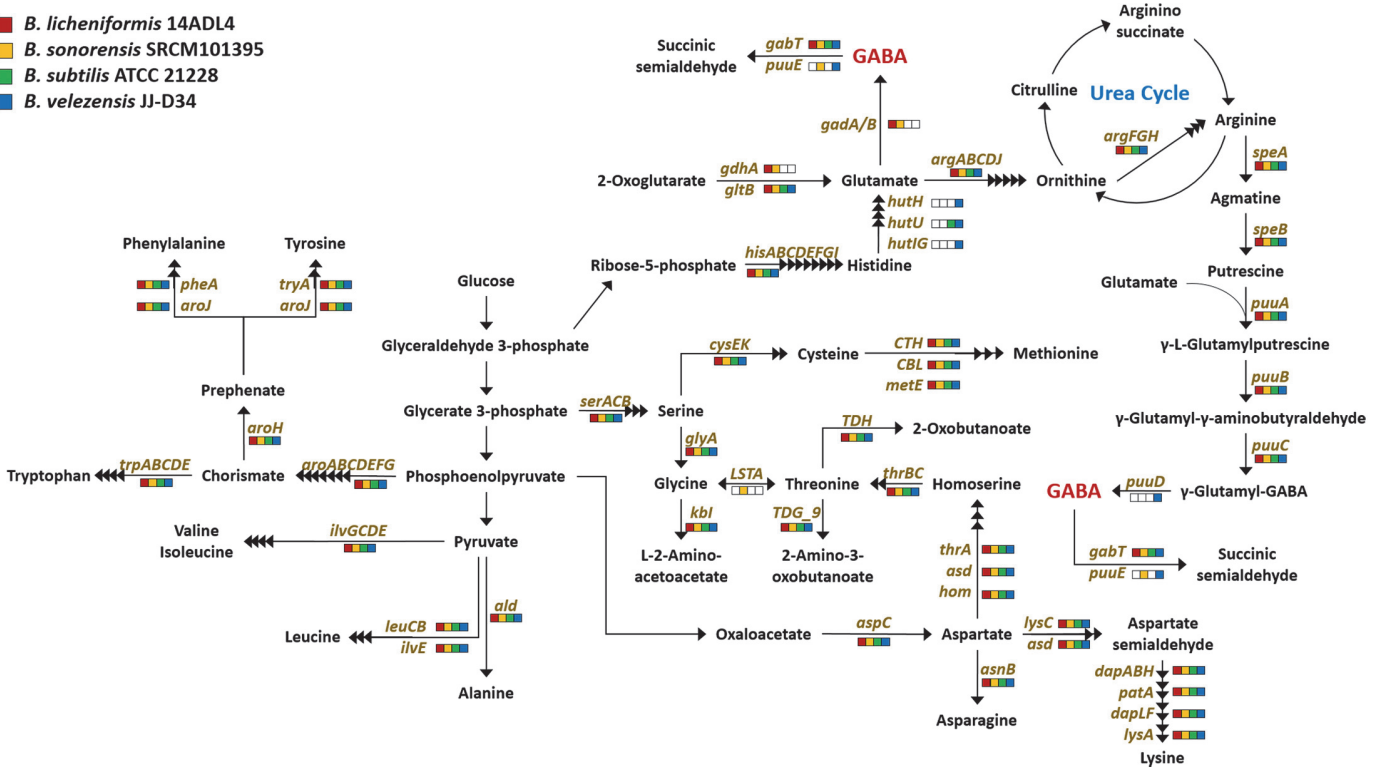

Fig. 4. Identification of genes involved in the amino acid metabolism of four Bacillus spp. strains.

Most of the amino acid metabolic pathway genes were identified in all four genomes (Fig. 4). However, genes involved in glutamate and arginine metabolism were not shared by all four strains. The B. licheniformis and $B$. sonorensis strain genomes contain homologs for the conversion of 2-oxoglutarate to GABA via glutamate, including a gad $A / B$ (glutamate decarboxylase) homolog encoding an enzyme that can convert glutamate to GABA [32]. Thus, the B. licheniformis and B. sonorensis strains may be able to produce GABA via this pathway. All four strains possess a $g a b T$ (GABA aminotransferase) homolog; this enzyme has been reported to decompose GABA into succinic semialdehyde [33]. The decrease of GABA in the soybean cultures of $B$. licheniformis and $B$. sonorensis can be attributed to their possession of gabT, but the metabolism in B. subtilis and B. velezensis is not clearly explained.

B. velezensis JJ-D34 has homologs for the conversion of histidine to glutamate, but the amount of glutamate identified in its soybean culture was the lowest among the soybean cultures of the four Bacillus spp. All four strains possess homologs of genes to produce $\gamma$-glutamyl- $\gamma$-aminobutyric acid ( $\gamma$-glutamyl-GABA) via putrescine from arginine, while only $B$. velezensis has a puuD ( $\gamma$-glutamyl- $\gamma$-aminobutyrate hydrolase) homolog that may convert $\gamma$-glutamyl-GABA to GABA [34]. The puuD homolog of B. velezensis strain JJ-D34 was annotated as a glutamine amidotransferase-encoding gene in the NCBI database (GenBank Accession No. AKF29542.1). A puuE (4aminobutyrate-2-oxoglutarate transaminase) homolog, encoding an enzyme known to convert GABA to succinic semialdehyde, was identified in the B. sonorensis and B. velezensis genomes [35]. The highest amount of GABA was identified in the B. velezensis cultures, indicating that the pathway for conversion of arginine to GABA was active, while the pathway for conversion of GABA to succinic semialdehyde was inactive. In the case of $B$. sonorensis SRCM101395, the annotated puuE is located between gabR (HTH-type transcriptional regulatory protein) and a gabT homolog and is smaller than puuE from B. velezensis JJ-D34 (513 bp compared with 1,269 bp) (Fig. 5). Additionally, deletions were identified in the gabR and gabT homologs of B. sonorensis SRCM101395 compared with the corresponding genes from the other strains. Further studies are required to show whether the puuE homologs of $B$. sonorensis and B. velezensis contribute to the conversion of GABA.

We could not find any clues as to whether the gabT homologs of B. subtilis and B. velezensis are involved in the conversion of GABA to succinic semialdehyde. We analyzed the flanking regions of the gabT homologs in the genomes of the four strains used in this study and found rocE (amino acid permease) homologs located downstream of the gabT homologs in the genomes of the B. licheniformis and B. sonorensis strains (Fig. 5). The $g a b T$ and rocE homologs form a putative operon with gabD (succinate-semialdehyde dehydrogenase). The expression of a GABA transporter gene might contribute to the decrease of GABA in the soybean cultures of B. licheniformis and B. sonorensis. B. subtilis ATCC 21228 possesses homologs of neither gadA/B nor puuD. The highest GABA production among the four Bacillus strains, by B. velezensis JJ-D34, may be attributed to its possession of puuD.

To determine whether the GABA-producing characteristics of the four Bacillus strains studied in this work can be extended to species-specific characteristics, we checked for the presence of five gene homologs supposedly involved in GABA metabolism in the complete genomes of many strains of the four Bacillus spp. (Table 3). Among 135 B. subtilis strains, only strain BEST7613 (AP012495.1) possesses an annotated gad, but its product has only $12.6 \%$ amino acid sequence homology with $B$. licheniformis DSM $13^{\mathrm{T}}$ glutamate decarboxylase (data not shown). Thus, the annotated gad of strain BEST7613 may not endow glutamate decarboxylase function. With few exceptions, we confirmed the species-specific presence of gadA/B, rocE, puuD, and puuE in the four Bacillus spp. 


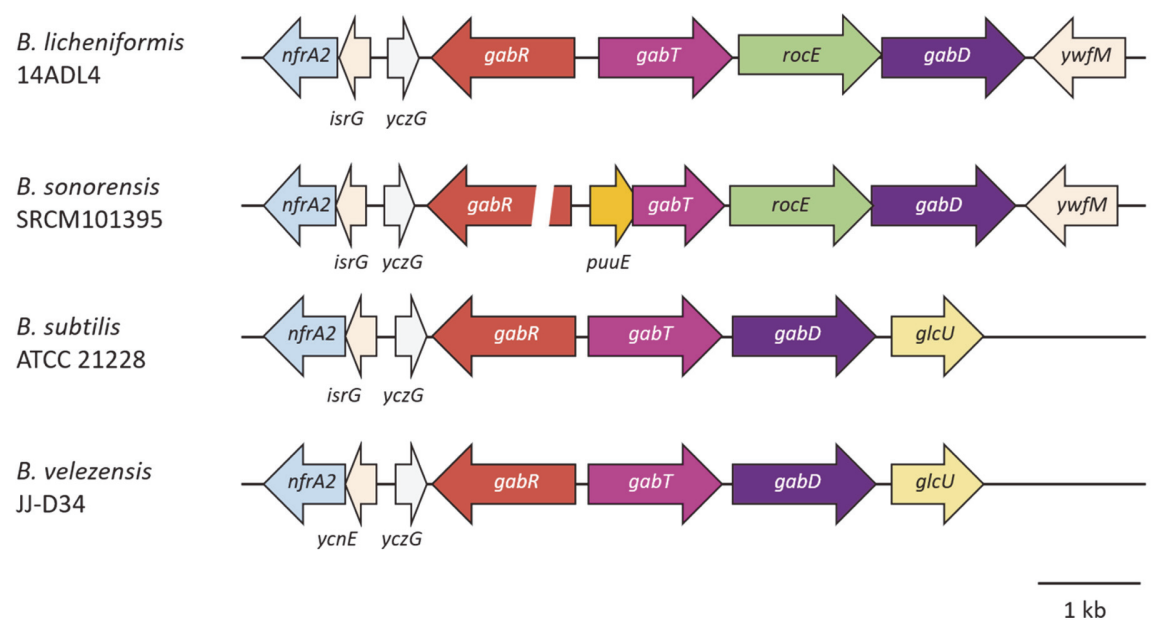

Fig. 5. Structures of genes surrounding the annotated $g a b T$ gene in four Bacillus genomes. Abbreviations: $g a b R$, HTH-type transcriptional regulatory protein-encoding gene; gabT, GABA aminotransferase-encoding gene; rocE, amino acid permease-encoding gene; $g a b D$, succinate-semialdehyde dehydrogenase-encoding gene.

Table 3. Presence of potential genes involved in GABA production (analysis of data available in March 2020).

\begin{tabular}{|c|c|c|c|c|c|c|}
\hline \multirow[b]{2}{*}{ Species } & \multicolumn{6}{|c|}{ Number of strains } \\
\hline & $\begin{array}{c}\text { Complete genomes } \\
\text { published }\end{array}$ & $\begin{array}{l}\operatorname{gad} A / B \\
\text { possessor }\end{array}$ & $\begin{array}{c}g a b T \\
\text { possessor }\end{array}$ & $\begin{array}{c}\text { rocE } \\
\text { possessor }\end{array}$ & $\begin{array}{c}\text { puuD } \\
\text { possessor }\end{array}$ & $\begin{array}{c}\text { puuE } \\
\text { possessor }\end{array}$ \\
\hline B. licheniformis & 27 & 27 & 27 & 27 & - & - \\
\hline B. sonorensis & 1 & 1 & 1 & 1 & - & (1) \\
\hline B. velezensis & 110 & - & 110 & - & 110 & 110 \\
\hline B. subtilis & 135 & (1) & 135 & - & - & 6 \\
\hline
\end{tabular}

Numbers in parentheses indicate the number of strains having the annotated genes and the genes may have a different function.

Among the 135 B. subtilis strains, six possess puuE homologs and their flanking regions exhibit similar gene structures to that in B. velezensis (data not shown). The presence of puuE homologs in the six B. subtilis strains might be the result of genetic recombination between B. subtilis and Bacillus strains possessing puuE homologs. The presence of $g a d A / B, r o c E$, and $p u u D$ can be used as a biomarker for the selection of GABA-producing starter culture candidates.

\section{Discussion}

Fermented foods are consumed all over the world. The application of starter cultures has been reported to provide technological, nutritional, and health advantages in terms of the final product composition. The first stage in designing a starter culture for a fermented food is to characterize the microbiota of the food matrix of interest and then select strains that are best suited to that environment. Traditionally, in starter selection, significant emphasis was placed on the technological phenotypes of strains, including growth performance or activity, flavor testing, and matrix formation analysis [36]. Sometimes, the selected strain does not reflect the true structure of the microbiota in the fermentation and fails to display adequate performance in the target fermentation process.

Traditional Bacillus-involved fermented soybean foods are consumed in many countries in Asia and Africa as a good source of protein, and B. subtilis has been known as the major fermenting species [37]. However, the progress of molecular taxonomy and next-generation sequencing technologies has created the need to shed new light on the major fermenters based on current taxonomic standards and their functional differences in soybean food fermentation, and will serve as a cornerstone for the selection of useful strains with target characteristics.

Soybeans are one of the most popular plant-based proteins used in food products, with a protein content of $35 \%-40 \%$ on a dry weight basis [38]. Therefore, proteolysis of soybean protein is one of the most important processes in terms of primary flavor development in soybean fermentation. Free amino acids have been reported to contribute directly to taste perception, and act as a precursor of flavor enhancement [39].

Several studies on the impact of fermentation on soybean protein have been performed and concluded that the profile of free amino acids in fermented products not only depends on the starter culture used, but also on the soybean variety used for fermentation [40]. In this study, we found differences in amino acid profiles of soybean cultures produced by strains of four Bacillus spp. and clues to the mechanisms of species-specific production through comparative genomic analysis. Our results will contribute to proper selection of Bacillus starter culture candidates for soybean food fermentation in accordance with the goals of each producer. B. licheniformis can be used to enhance the concentration of glutamic acid, which is a key compound that determines umami taste of fermented soybean foods including soy sauce [41]. B. subtilis has good potential to increase the concentrations of 
the essential amino acids isoleucine, leucine, methionine, phenylalanine, tryptophan, valine, and lysine in fermented soybean foods. B. velezensis was shown to produce large amounts of GABA, a well-known bioactive compound that increases the value of fermented soybean products. Further metabolic studies with multiple strains of the four Bacillus spp. are required to prove that the results of this study can be extended to the characteristics of each species.

\section{Acknowledgments}

This work was supported by the National Research Foundation of Korea (NRF) [NRF-2016R1D1A1B01011421 and NRF-2019R1A2C1003639]. Haram Kong was supported by Kyonggi University's Graduate Research Assistantship 2020. We thank Dr. Jochen Blom at Justus-Liebig University for EDGAR analysis. We thank James Allen, DPhil, from Edanz Group (https://en-author-services.edanzgroup.com/) for editing a draft of this manuscript.

\section{Conflict of Interest}

The authors have no financial conflicts of interest to declare.

\section{References}

1. Park KY, Hwang KM, Jung KO, Lee KB. 2002. Studies on the standardization of doenjang (Korean soybean paste). J. Korean Soc. Food Sci. Nutr. 31: 343-350.

2. Shin D, Jeong D. 2015. Korean traditional fermented soybean products: Jang. J. Ethnic Foods 2: 2-7.

3. Choi KK, Chi CB, Ham SS, Lee DS. 2003. Isolation, identification and growth characteristics of main strain related to meju fermentation. J. Korean Soc. Food Sci. Nutr. 32: 818-824.

4. Kang MJ, Kim SH, Joo HK, Lee GS, Yim MH. 2000. Isolation and identification of microorganisms producing the soy protein hydrolyzing enzyme from traditional mejus. J. Korean Soc. Agric. Chem. Biotechnol. 43: 86-94.

5. Kwon OJ, Kim JK, Chung YK. 1986. The characteristics of bacteria isolated from ordinary Korean soy sauce and soybean paste. J. Korean Agri. Chem. Soc. 29: 422-428.

6. Yoo SK, Cho WH, Kang SM, Lee SH. 1999. Isolation and identification of microorganisms in Korean traditional soybean paste and soybean sauce. Korean J. Appl. Microbiol. Biotechnol. 27: 113-117.

7. Cho KM, Seo WT. 2007. Bacterial diversity in Korean traditional soybean fermented foods (doenjang and ganjang) by $16 \mathrm{~S}$ rRNA gene sequence analysis. Food Sci. Biotechnol. 16: 320-324.

8. Jung JY, Lee SH, Jeon CO. 2014. Microbial community dynamics during fermentation of doenjang-meju, traditional Korean fermented soybean. Int. J. Food Microbiol. 185: 112-120.

9. Jung WY, Jung JY, Lee HJ, Jeon CO. 2016. Functional characterization of bacterial communities responsible for fermentation of doenjang: a traditional Korean fermented soybean paste. Front. Microbiol. 7: 827.

10. Kim TW, Lee JH, Kim SE, Park MH, Chang HC, Kim HY. 2009. Analysis of microbial communities in doenjang, a Korean fermented soybean paste, using nested PCR-denaturing gradient gel electrophoresis. Int. J. Food Microbiol. 131: 265-271.

11. Kim YS, Jeong DY, Hwang YT, Uhm TB. 2011. Bacterial community profiling during the manufacturing process of traditional soybean paste by pyrosequencing method. Korean J. Microbiol. 47: 275-280.

12. Kim YS, Kim MC, Kwon SW, Kim SJ, Park IC, Ka JO, et al. 2011. Analyses of bacterial communities in meju, a Korean traditional fermented soybean bricks, by cultivation-based and pyrosequencing methods. J. Microbiol. 49: 340-348.

13. Lee JH, Kim TW, Lee H, Chang HC, Kim HY. 2010. Determination of microbial diversity in meju, fermented, cooked soya beans, using nested PCR-denaturing gradient gel electrophoresis. Lett. Appl. Microbiol. 51: 388-394.

14. Lee SY, Kim HY, Lee S, Lee JM, Muthaiya MJ, Kim BS, et al. 2012. Mass spectrometry-based metabolite profiling and bacterial diversity characterization of Korean traditional meju during fermentation. J. Microbiol. Biotechnol. 22: 1523-1531.

15. Nam YD, Lee SY, Lim SI. 2012. Microbial community analysis of Korean soybean pastes by next-generation sequencing. Int. J. Food Microbiol. 155: 36-42.

16. Franco-Duarte R, Černáková L, Kadam S, Kaushik KS, Salehi B, Bevilacqua A, et al. 2019. Advances in chemical and biological methods to identify microorganisms - from past to present. Microorganisms 7: 130.

17. Dunlap CA, Kwon SW, Rooney AP, Kim, SJ. 2015. Bacillus paralicheniformis sp. nov., isolated from fermented soybean paste. Int. J. Syst. Evol. Microbiol. 65: 3487-3492.

18. Dunlap CA, Kim SJ, Kwon SW, Rooney AP. 2016. Bacillus velezensis is not a later heterotypic synonym of Bacillus amyloliquefaciens; Bacillus methylotrophicus, Bacillus amyloliquefaciens subsp. plantarum and 'Bacillus oryzicola' are later heterotypic synonyms of Bacillus velezensis based on phylogenomics. Int. J. Syst. Evol. Microbiol. 66: 1212-1217.

19. Fan B, Blom J, Klenk HP, Borriss R. 2017. Bacillus amyloliquefaciens, Bacillus velezensis, and Bacillus siamensis form an "Operational Group B. amyloliquefaciens" within the B. subtilis species complex. Front. Microbiol. 8: 22.

20. Jang M, Jeong DW, Lee JH. 2019. Identification of the predominant Bacillus, Enterococcus, and Staphylococcus species in meju, a spontaneously fermented soybean product. Microbiol. Biotechnol. Lett. 47: 1-5.

21. Chang M, Chang HC. 2007. Characteristics of bacterial-koji and doenjang (soybean paste) made by using Bacillus subtilis DJI. Korean J. Microbiol. Biotechnol. 35: 325-333.

22. Cho MJ, Shim JM, Lee JY, Lee KW, Yao Z, Liu X, et al. 2016. Properties of meju fermented with multiple starters. Microbiol. Biotechnol. Lett. 44: 109-116.

23. Hong Y, Jung HJ, Han SK, Kim HY. 2016. Potentiality of Bacillus amyloliquefaciens KFCC11574P isolated from Korean traditional doenjang as a starter in the production of functional soya bean paste. Int. J. Food Sci. Technol. 51: 105-113.

24. Ji WD, Yang SH, Choi MR, Kim JK. 1995. Volatile components of Korean soybean paste produced by Bacillus subtilis PM3. J. Microbiol. Biotechnol. 5: 143-148.

25. Lee KH, Choi HS, Hwang KA, Song J. 2016. Quality changes in doenjang upon fermentation with two different Bacillus subtilis strains. J. East Asian Soc. Diet. Life 26: 163-170.

26. Yoo SK, Kang SM, Noh YS. 2000. Quality properties on soy bean pastes made with microorganisms isolated from traditional soy bean paste. Korean J. Food Sci. Technol. 32: 1266-1270.

27. Jeong DW, Lee B, Lee JH. 2018. Complete genome sequence of Bacillus licheniformis 14ADL4 exhibiting resistance to clindamycin. Korean J. Microbiol. 54: 169-170.

28. Jung JY, Chun BH, Moon JY, Yeo SH, Jeon CO. 2016. Complete genome sequence of Bacillus methylotrophicus JJ-D34 isolated from deonjang, a Korean traditional fermented soybean paste. J. Biotechnol. 219: 36-37. 
29. Blom J, Albaum SP, Doppmeier D, Puhler A, Vorholter F-J, Zakrzewski M, et al. 2009. EDGAR: a software framework for the comparative analysis of prokaryotic genomes. BMC Bioinformatics 10: 154 .

30. Aziz RK, Bartels D, Best AA, Dejongh M, Disz T, Edwards RA, et al. 2008. The RAST Server: rapid annotations using subsystems technology. BMC Genomics 9: 75.

31. Jeong DW, Heo S, Lee B, Lee H, Jeong K, Her JY, et al. 2017. Effects of the predominant bacteria from meju and doenjang on the production of volatile compounds during soybean fermentation. Int. J. Food Microbiol. 262: 8-13.

32. Park KB, Oh SH. 2006. Enhancement of $\gamma$-aminobutyric acid production in Chungkukjang by applying a Bacillus subtilis strain expressing glutamate decarboxylase from Lactobacillus brevis. Biotechnol. Lett. 28: 1459-1463.

33. Le Vo TD, Kim TW, Hong SH. 2012. Effects of glutamate decarboxylase and gamma-aminobutyric acid (GABA) transporter on the bioconversion of GABA in engineered Escherichia coli. Bioprocess Biosyst. Eng. 35: 645-650.

34. Kurihara S, Oda S, Kumagai H, Suzuki H. 2006. $\gamma$-Glutamyl- $\gamma$-aminobutyrate hydrolase in the putrescine utilization pathway of Escherichia coli K-12. FEMS Microbiol. Lett. 256: 318-323.

35. Kurihara S, Kato K, Asada K, Kumagai H, Suzuki H. 2010. A putrescine-inducible pathway comprising PuuE-YneI in which gammaaminobutyrate is degraded into succinate in Escherichia coli K-12. J. Bacteriol. 192: 4582-4591.

36. Ammor MS, Mayo B. 2007. Selection criteria for lactic acid bacteria to be used as functional starter cultures in dry sausage production: An update. Meat Sci. 76: 138-146.

37. Tamang JP, Watanabe K, Holzapfel WH. 2016. Review: diversity of microorganisms in global fermented foods and beverages. Front. Microbiol. 7: 377

38. Mujoo R, Trinh DT, Ng PKW. 2003. Characterization of storage proteins in different soybean varieties and their relationship to tofu yield and texture. Food Chem. 82: 265-273.

39. Qin L, Ding X. 2007. Formation of taste and odor compounds during preparation of Douchiba, a Chinese traditional soy-fermented appetizer. J. Food Biochem. 31: 230-251.

40. Sanjukta S, Rai AK. 2016. Production of bioactive peptides during soybean fermentation and their potential health benefits. Trends Food Sci. Technol. 50: 1-10.

41. Lioe HN, Selamat J, Yasuda M. 2010. Soy sauce and its umami taste: a link from the past to current situation. J. Food Sci. 75: R71-R76. 